\title{
DETERMINAÇÃO DO PONTO DE NÉVOA EM SURFACTANTES NÃO IÔNICOS POR ESPECTROSCOPIA DE IMPEDÂNCIA ELÉTRICA
}

\author{
Sandro Vagner de Lima e Helinando Pequeno de Oliveira* \\ Colegiado de Pós-Graduação em Ciência dos Materiais, Universidade Federal do Vale do São Francisco, 48902-300 Juazeiro - \\ BA, Brasil
}

Recebido em 31/3/10; aceito em 2/6/10; publicado na web em 1/9/10

\begin{abstract}
CLOUD POINT IN NON-IONIC SURFACTANTS PROBED BY ELECTRICAL IMPEDANCE SPECTROSCOPY. In this paper, we analyze the use of electrical impedance spectroscopy applied to determination of cloud point. The slope of admittance measured at $100 \mathrm{kHz}$ is reduced to temperature above the critical value which characterizes the phase transition, in a strong indication that this process is activated during the clouding. Additionally to this study we explored the influence of parameters such as additives and temperature on the performance of phase separation of residues (silver nanoparticles) by cloud point extraction. The interaction with salt maximizes the separation of chemical residues in a progressively reduced temperature.
\end{abstract}

Keywords: electrical impedance spectroscopy; cloud point; non-ionic surfactants.

\section{INTRODUÇÃO}

Surfactantes são estruturas anfifílicas constituídas por partes que apresentam afinidades distintas em ambientes específicos. A agregação destas moléculas se dá pela interação entre as porções hidrofóbicas, minimizando a interação com a água. Surfactantes não iônicos compostos por grupos de polietileno óxido (PEO) (como no caso do Triton X-100, usado neste trabalho) são caracterizados pela forte dependência da solubilidade com a temperatura, uma vez que hidratação das cadeias de PEO diminui com o aumento na agitação térmica. ${ }^{1}$

Neste sistema, a redução da solubilidade varia com a permissividade dielétrica de solvente. ${ }^{2}$ A instabilidade observada nas micelas é promovida pela desidratação de grupos hidrofílicos com a consequente formação de micelas gigantes (resultado da atração intermicelar) proporcionando reduzida solubilidade em água, o que define a transição de ponto de névoa. ${ }^{3}$

Esse fenômeno é caracterizado pela estruturação de duas fases: uma delas é rica em surfactante (fase coacervada) e a outra contém surfactante com concentração próxima à concentração micelar crítica (CMC) - definida como fase diluída. ${ }^{4,5} \mathrm{O}$ volume da fase coacervada é relativamente menor que o volume da fase diluída, o que contribui para o acúmulo de surfactante na região do precipitado. É importante lembrar que essa propriedade não se restringe apenas a surfactantes, sendo também aplicável a drogas com caráter anfifílico, causando a agregação de moléculas, o que vem a alterar a atividade bioquímica das mesmas. ${ }^{6-11}$

Vários estudos têm sido desenvolvidos na direção de determinar o ponto de névoa em moléculas com caráter anfifílico, assim como o efeito de aditivos sobre essa transição. ${ }^{12-15}$ Essa propriedade de surfactantes não iônicos é de grande interesse prático devido ao uso corrente do método de extração por ponto de névoa, permitindo várias aplicações das quais podemos destacar: a extração de resíduos químicos de ambiente aquoso e de proteínas do sangue. ${ }^{16}$

O procedimento padrão utilizado para detectar a formação das nuvens em solução aquosa é a visualização direta. ${ }^{17} \mathrm{Com}$ esse procedimento, a análise se restringe à determinação da temperatura crítica na qual ocorre a separação de fases do coloide.
Neste trabalho propomos o uso de uma ferramenta não convencional, a espectroscopia de impedância elétrica (EIS), com potencial utilidade não apenas na determinação do ponto de névoa, mas também na caracterização dos processos de distribuição de agregados micelares em fase aquosa, com a medida dos parâmetros tais como corrente e polarização interfaciais, uma vez que a formação de uma fase coacervada permite com que a fase complementar seja constituída por uma larga distribuição de agregados de menor diâmetro. Como consequência, se tem aumento na difusibilidade dos mesmos, vindo a afetar consequentemente a resposta elétrica do sistema. Diversos trabalhos voltados para a análise de sistemas coloidais mostram a potencialidade dessa técnica no mapeamento de transições de fase, principalmente pelo fato de ser possível separar fenômenos que acontecem na interface eletrodo/eletrólito daqueles que ocorrem no volume da solução (formação de dupla camada elétrica e efeitos volumétricos interfaciais), o que torna a técnica de EIS aplicável neste estudo. ${ }^{18-23}$

Além da caracterização elétrica, a análise fatorial de conjuntos de $2^{4}$ experimentos foi desenvolvida com o objetivo de verificar o efeito dos fatores selecionados na eficiência de extração de resíduos de prata em solução aquosa, a partir do método de extração por ponto de névoa. Dessa forma, podemos dividir o trabalho em duas partes: a caracterização elétrica do fenômeno de ponto de névoa e o estudo quimiométrico para otimização do processo de extração de contaminantes (coloide de prata) por esse processo, extremizando os parâmetros: concentração de surfactante não iônico, presença de sal (cloreto de sódio), concentração de surfactante iônico (SDS) e temperatura.

\section{PARTE EXPERIMENTAL}

\section{Materiais}

Triton X-100 (TX -100), triton X-405 (TX-405), tween 80 (TW-80), dodecil sulfato de sódio (SDS), citrato de sódio, nitrato de prata e cloreto de sódio foram adquiridos junto à Aldrich (EUA) e usados como recebidos, sem purificação adicional. Água ultrapura $(\mathrm{R}=18,2 \mathrm{M} \Omega$ ) foi obtida por osmose reversa com o uso do sistema NANOpure (EUA). 


\section{Preparação das amostras}

Todas as amostras foram preparadas usando a concentração de 10 mg/mL de surfactante não iônico (TX-100, TX-405 e TW- 80). O coloide de prata utilizado como rejeito a ser eliminado da solução aquosa foi preparado conforme procedimento descrito na literatura. ${ }^{24}$

Inicialmente foram introduzidos $90 \mathrm{mg}$ de nitrato de prata em 500 $\mathrm{mL}$ de água, mantida à temperatura de $90^{\circ} \mathrm{C}$. Em seguida, $10 \mathrm{~mL}$ de solução com $1 \%$ em massa de citrato de sódio foram colocados na solução de nitrato de prata. O processo de síntese leva aproximadamente $2 \mathrm{~h}$, desde que o coloide seja mantido sob intensa agitação a $90^{\circ} \mathrm{C}$.

\section{Estudo quimiométrico}

Os fatores analisados foram extremizados assumindo os seguintes valores: triton X-100 (30 e $60 \mathrm{mg} / \mathrm{mL}), \mathrm{SDS}$ (0 e $20 \mathrm{mM}), \mathrm{NaCl}(0$ e $100 \mathrm{mM})$, temperatura $\left(70\right.$ e $\left.85^{\circ} \mathrm{C}\right)$, considerados como valores mínimos (-) e máximos (+), respectivamente. O triton X-100 foi escolhido para esse estudo por apresentar o mais baixo ponto de névoa entre todos os surfactantes estudados. Usando todas as possibilidades de combinação entre os parâmetros verificamos que foi necessária a realização de rodadas de 16 experimentos. Na Tabela 1 são especificadas as amostras preparadas e seus códigos.

Tabela 1. Descrição das amostras preparadas no planejamento fatorial

\begin{tabular}{|c|c|c|c|c|c|}
\hline Amostra & Temperatura & {$[\mathrm{NaCl}]$} & [TX-100] & [SDS] & Códigos \\
\hline 1 & - & - & - & - & I \\
\hline 2 & - & - & - & + & $\mathrm{D}$ \\
\hline 3 & - & - & + & - & $\mathrm{C}$ \\
\hline 4 & - & + & - & - & B \\
\hline 5 & - & - & + & + & $\mathrm{CD}$ \\
\hline 6 & - & + & - & + & $\mathrm{BD}$ \\
\hline 7 & - & + & + & - & $\mathrm{BC}$ \\
\hline 8 & - & + & + & + & $\mathrm{BCD}$ \\
\hline 9 & + & - & - & - & A \\
\hline 10 & + & - & - & + & $\mathrm{AD}$ \\
\hline 11 & + & - & + & - & $\mathrm{AC}$ \\
\hline 12 & + & + & - & - & $\mathrm{AB}$ \\
\hline 13 & + & - & + & + & $\mathrm{ACD}$ \\
\hline 14 & + & + & - & + & $\mathrm{ABD}$ \\
\hline 15 & + & + & + & - & $\mathrm{ABC}$ \\
\hline 16 & + & + & + & + & $\mathrm{ABCD}$ \\
\hline
\end{tabular}

\section{Métodos experimentais}

A resposta elétrica das soluções foi obtida a partir do uso do analisador de impedância Solartron 1260 (Reino Unido) e por um medidor LCR Agilent 4263B (EUA). Para determinar a temperatura de névoa procedemos com a introdução das amostras em um banho térmico, promovendo o aquecimento sob condição de intensa agitação. Para a análise da resposta elétrica como função da frequência, o potencial aplicado foi fixado em $10 \mathrm{mV}$ (sem polarização externa) em uma faixa de frequência variável de $1 \mathrm{~Hz}$ a $1 \mathrm{MHz}$. Em cada experimento, duas placas de aço inoxidável com dimensões de $20 \times 5 \mathrm{~mm}^{2}$ dispostas paralelamente a uma distância de $17 \mathrm{~mm}$ foram colocadas dentro de um béquer de $25 \mathrm{~mL}$ contendo $20 \mathrm{~mL}$ da solução de interesse. A análise dos dados experimentais foi realizada através do programa Zview (Solartron, Reino Unido).
O tamanho das partículas foi determinado usando o medidor de tamanho de partículas Zeta Sizer Nano Z590 (Reino Unido), enquanto que os espectros de absorção na região do UV-vis foram levantados usando o espectrofotômetro DR-5000 (Hach, Alemanha) com comprimento de onda na faixa de $180-800 \mathrm{~nm}$, sendo o pico característico da prata o parâmetro usado para definir a concentração do contaminante em solução.

\section{Modelagem (Método de Maxwell-Wagner)}

A presença de estruturas mesoscópicas em solução aquosa promove um considerável aumento na razão superfície/volume e, consequentemente, nos efeitos interfaciais. Com isso, se tem a formação de uma dupla camada elétrica na interface eletrólito/eletrodo e polarização interfacial no volume descrito por Maxwell-Wagner. ${ }^{25-27}$

A equação de Maxwell-Wagner descreve a relação entre a permissividade complexa do sistema $\left(\varepsilon^{*}\right)$, das partículas dispersas $\left(\varepsilon_{p}\right)$ e do meio $\left(\varepsilon_{\mathrm{A}}^{*}\right)$ de acordo com a Equação 1:

$$
\frac{\varepsilon^{*}-\varepsilon_{A}^{*}}{\varepsilon^{*}-2 \varepsilon_{A}^{*}}=\frac{\varepsilon_{P}^{*}-\varepsilon_{A}^{*}}{\varepsilon_{P}^{*}-2 \varepsilon_{A}^{*}} \cdot \phi
$$

sendo $\phi$ a fração volumétrica de uma suspensão de partículas esféricas em uma solução randômica diluída.

O processo de relaxação interfacial descrito por Maxwell-Wagner ${ }^{28}$ é caracterizado pela circulação de corrente na interface entre dois ou mais dielétricos diferentes. A resposta dielétrica da interface é representada por uma associação em série de circuitos R-C em paralelo, ${ }^{29}$ com impedância resultante dada pela Equação 2:

$$
Z=\sum_{i=1}^{n} Z_{i}=\sum_{i=1}^{n} \frac{R_{i}}{1+\omega^{2} \tau_{i}^{2}}-j \omega \sum_{i=1}^{n} \frac{\tau_{i} R_{i}}{1+\omega^{2} \tau_{i}^{2}}
$$

sendo $\tau_{i}$ o tempo característico definido para cada par de resistor e capacitor $\tau_{i}=R_{i} C_{i}$.

\section{RESULTADOS E DISCUSSÃO}

\section{Ponto de névoa}

Os resultados obtidos indicam que a resposta elétrica das soluções aquosas contendo surfactante a diferentes temperaturas é caracterizada pela existência de duas respostas lineares com derivadas distintas, sendo a temperatura em que ocorre a interseção entre as retas definida como o ponto de névoa.

Como podemos ver na Figura 1, para temperaturas inferiores ao valor crítico (que define a transição visual do ponto de névoa), a admitância da solução cresce com a temperatura a uma taxa relativamente alta caracterizando a formação progressiva de micelas gigantes, reduzindo o livre caminho médio para o transporte de carga. Esse comportamento se mostra universal para todas as amostras analisadas, o que nos permite associar a quebra na derivada com a existência do mecanismo induzido pela transição de fase (ponto de névoa) similarmente ao que é verificado em processos do tipo CMC, CAC e ponto Krafft, dada a boa concordância entre os valores obtidos com aqueles verificados por inspeção visual. A redução abrupta na derivada da admitância versus temperatura indica que a taxa de formação de agregados em solução é diminuída quando da ocorrência do ponto de névoa, uma vez que com a transição de fase se tem a instabilidade das micelas gigantes, que passam a precipitar em solução, contribuindo para a separação de fases e a obtenção de 


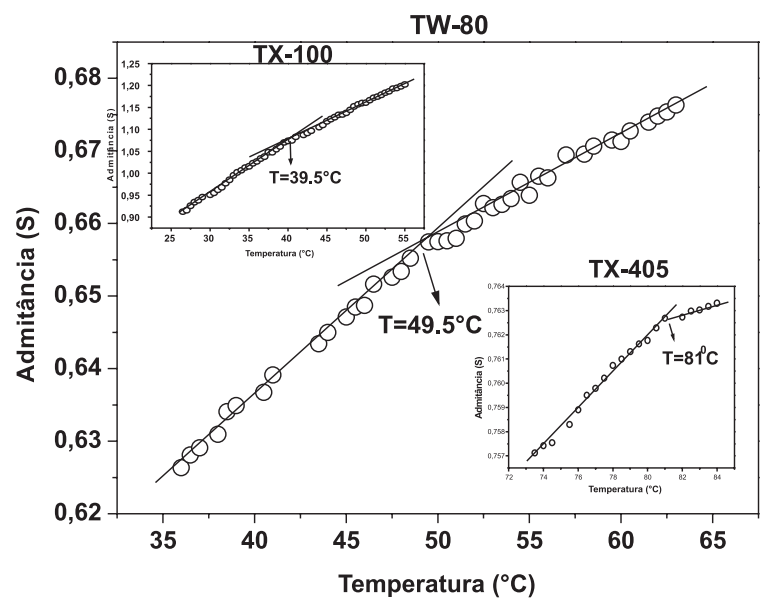

Figura 1. Curvas de admitância versus temperatura para os sistemas TX$100+[\mathrm{NaCl}]=1,5 \mathrm{M}, \mathrm{TW}-80+[\mathrm{NaCl}]=2,5 \mathrm{Me} \mathrm{TX}-405+[\mathrm{NaCl}]=1,5 \mathrm{M}$

um novo rearranjo de micelas em solução com diâmetro inferior às partículas depositadas na fase coacervada.

Um dos grandes interesses da indústria química é o de promover a adequada separação de fases a temperaturas consideravelmente reduzidas. O uso da técnica de detecção do ponto de névoa por EIS permite com que tenhamos o mapeamento direto da influência do sal sobre o ponto de névoa dos surfactantes de interesse, como mostra a Figura 2.

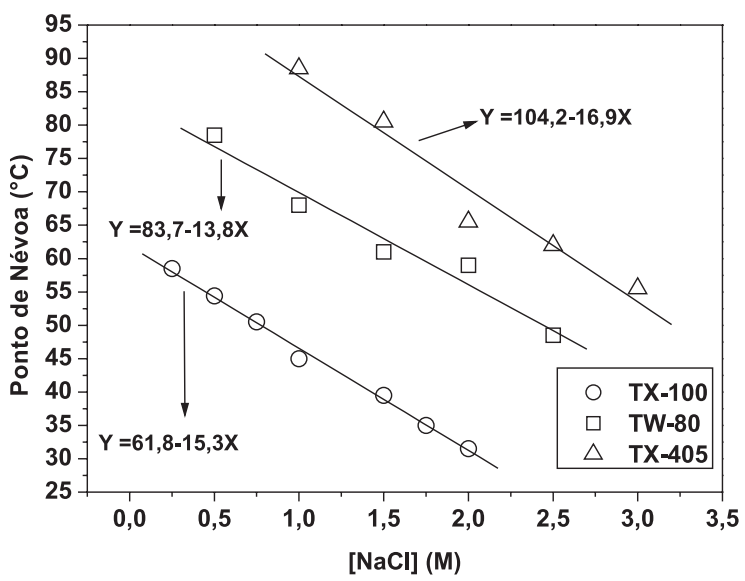

Figura 2. Temperatura do ponto de névoa versus concentração de cloreto de sódio para os sistemas analisados

Como podemos perceber, a introdução de $\mathrm{NaCl}$ nas soluções promove a redução da temperatura característica da transição de fase, com a eficiência de redução da temperatura de ponto de névoa medida diretamente pelo coeficiente angular das curvas na Figura 2, fornecendo a ordem TX-405>TX-100>TW-80.

Note que o TX-100 associa a mais baixa temperatura para qualquer concentração de sal, o que potencializa a sua aplicação como agente removedor de resíduos químicos, justificando a sua escolha dentro do conjunto de surfactantes estudados para aplicação no planejamento fatorial.

A resposta elétrica completa da solução aquosa com TX-100 à temperatura ambiente é mostrada na Figura 3. A dispersão de surfactante não iônico em água tem resposta elétrica reproduzida a partir de dois circuitos RC paralelos dispostos em série com frequência de relaxação em 3,74 e 6,02 MHz, respectivamente. Após a separação de fases induzida pelo ponto de névoa é observado o deslocamento nas frequências de relaxação dos processos interfaciais para 5,15 e

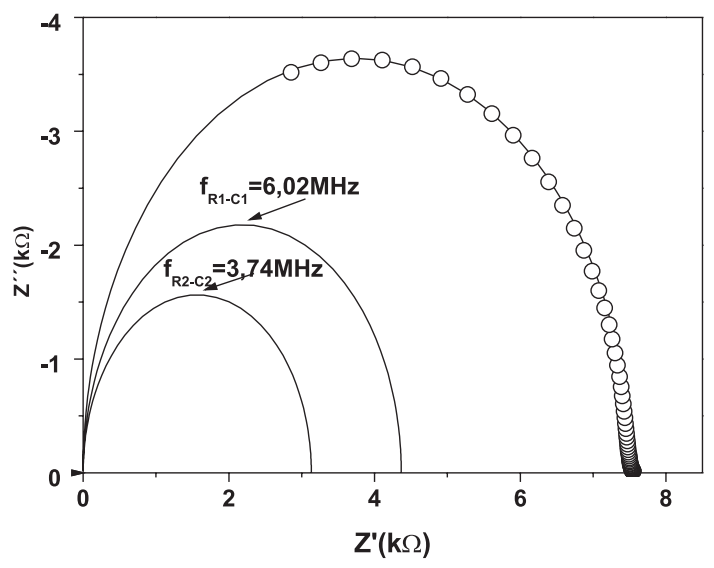

Figura 3. Diagrama de Nyquist para solução virgem contendo TX-100 (sem tratamento térmico) - pontos experimentais e ajuste teórico (linhas) referente à resposta de dois circuitos $R$ - $C$ (isolados e de forma combinada), reproduzindo a resposta do sistema

69,54 MHz caracterizando a retenção das partículas na fase coacervada, o que possibilita que as partículas dispersas em solução sejam menores do que na solução virgem, como comprovado por medidas de tamanho de partículas, com o tamanho médio na fase superior reduzida de 229,30 para 70,89 nm após tratamento térmico.

Com a introdução do coloide de prata podemos visualizar diretamente o processo de precipitação do coloide pela transparência de fase superior, como mostram as imagens da Figura 4.

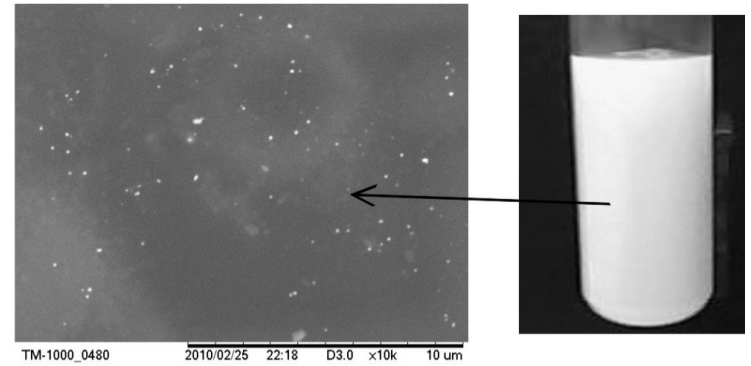

Antes

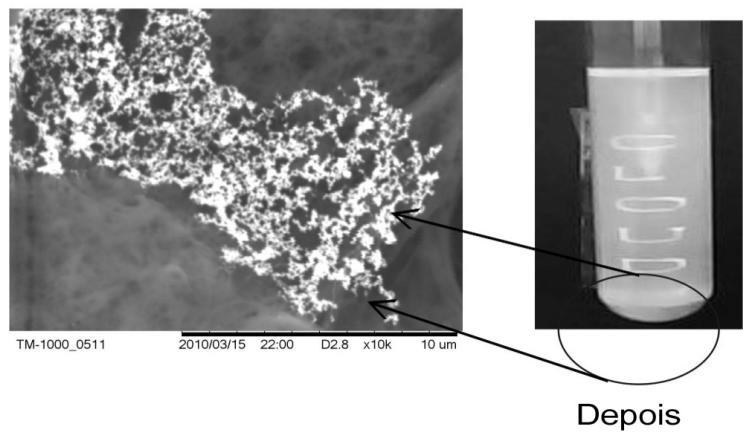

Figura 4. Microscopia eletrônica da fase diluída e concentrada das soluções aquosas contendo nanopartículas de prata (antes e após o processo de ponto de névoa)

Note que nesse caso as partículas metálicas inicialmente distribuídas uniformemente (na microscopia apresentada no lado esquerdo da Figura 4) passam a apresentar um alto grau de agregação após o tratamento térmico.

Com o intuito de medir a eficiência de extração desses resíduos da solução aquosa utilizamos como parâmetro de análise a intensi- 
dade de absorção na região do UV-vis do elemento a ser extraído da solução. Dessa forma, definimos como resposta do planejamento fatorial a eficiência de eliminação de nanopartículas de prata da solução, determinada diretamente pela relação $\left(A_{\text {antes }}-A_{\text {depois }}\right) / A_{\text {antes }}$, onde $A_{\text {antes }}$ representa a intensidade do pico de absorção do contaminante antes do aquecimento e $A_{\text {depois }}$ caracteriza a absorção da fase diluída no pico do contaminante após o aquecimento (para o caso da prata $\lambda=476 \mathrm{~nm}$ ). Note que com essa definição utilizamos uma variável com limites de 0 (nenhuma extração de rejeito) e 1 (máxima extração) levando em conta a aplicação de Lambert-Beer que relaciona intensidade de absorção com concentração da molécula no meio.

Fazendo uso dessa relação, diferentes amostras foram preparadas como forma de otimizar a extração de prata da solução. Neste sentido, a escolha foi feita pela amostra com pior rendimento considerando o procedimento padrão de extração $\left(85^{\circ} \mathrm{C}\right.$ e ausência de aditivos $)$. Dos experimentos realizados, verificamos que a amostra com pior rendimento continha $25 \%$ em massa de prata, sendo essa a concentração padrão a ser otimizada nos experimentos. Para simplificar a análise da importância dos parâmetros calculamos os fatores considerando a possibilidade de combinação de 3 parâmetros calculados para a condição de extremos $(-$ e + ) em um quarto fator.

Como podemos ver na Figura 5, a temperatura representa o fator de maior importância na extração dos resíduos, em especial na ausência de aditivos (amostra I) e com o aumento na concentração do surfactante não iônico (amostra C). Por outro lado, a amostra CD (máximo de SDS e TX-100) tem eficiência nula de remoção para qualquer das temperaturas trabalhadas, em uma forte indicação de que ocorre interação entre as partes hidrofóbicas de SDS e TX-100. Em todos os outros casos foi observada elevação da resposta com o aumento na temperatura, reforçando a hipótese de que nessa situação se tem uma forte redução na constante dielétrica do meio, afetando substancialmente a solubilidade dos grupos de PEO dispersos em solução.

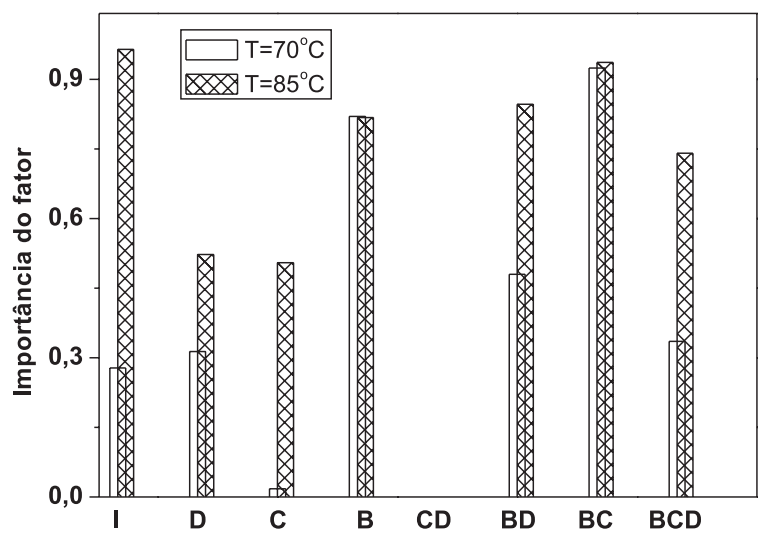

Figura 5. Importância do fator temperatura na extração da prata em solução aquosa

Como esperado, a introdução de sal na solução exerce papel de fundamental importância na redução da temperatura crítica para o ponto de névoa. É importante perceber na Figura 6 que a eficiência de extração da amostra com máxima concentração de sal é bem maior do que a observada na concentração mínima (amostra C e CD), indicando que a interação com máxima concentração de TX-100 passa a ser a condição que otimiza a extração de rejeitos da solução aquosa.

Relativamente à introdução de uma segunda classe de moléculas anfifílicas assumidas como aditivo (SDS) foi possível verificar que a interação com o TX-100 reduz significativamente a eficiência de remoção das nanopartículas, uma vez que nessa

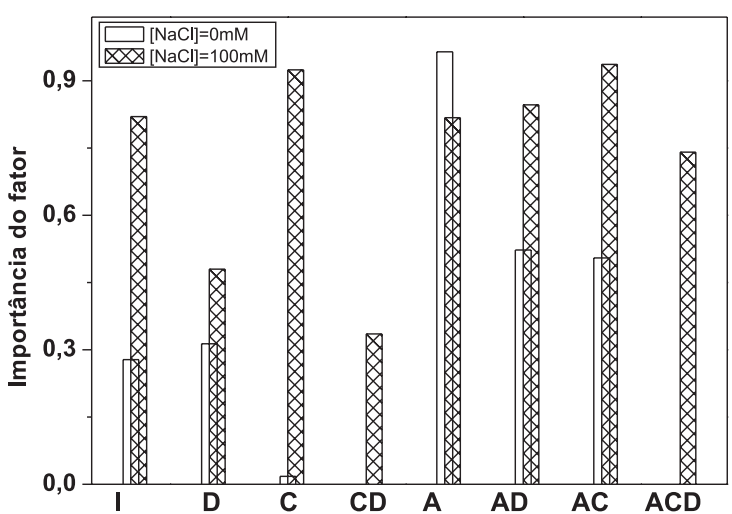

Figura 6. Importância do fator concentração de sal na extração da prata em solução aquosa

situação passa a ocorrer forte interação entre as moléculas de SDS e TX-100, reduzindo a extração das nanopartículas de prata a partir da solução aquosa.

\section{CONCLUSÕES}

Os processos interfaciais decorrentes da aplicação de um potencial elétrico em coloides permitem que se tenha a adequada descrição dos mecanismos de separação de fase por ponto de névoa. $\mathrm{O}$ uso da espectroscopia de impedância permite que se tenha um entendimento microscópico dos processos que conduzem à separação de fases e consequente remoção de resíduos químicos. A adequada escolha dos aditivos permite que a eficiência de remoção seja otimizada, o que é refletido diretamente nos parâmetros físicoquímicos medidos por absorção no UV-vis e por EIS. A interação entre moléculas de anfifílicos diferentes promove o processo de interação intermicelar, refletido na redução da remoção de resíduos químicos da solução aquosa.

\section{REFERÊNCIAS}

1. Sharma, K. S.; Patil, S. R.; Rakshit, A. K.; Colloids Surf., A 2003, 219, 67.

2. Huang, Y.; Qin, W.; Huo, X. P.; Dai, Y. Y.; Colloids Surf., A 2006, 276, 228.

3. Akbas, H.; Boz, M.; Batigoc, C.; Spectrochim. Acta, Part A 2010, 75, 671.

4. Li, J. L.; Bai, D. S.; Chen, B. H.; Colloids Surf., A 2009, 346, 237.

5. Taechangam, P.; Scamehorn, J. F.; Osuwan, S.; Rirksomboon, T.; Colloids Surf., A 2009, 347, 200.

6. Kim, E. J.; Shah, D. O.; Abstr. Paper Am. Chem. Soc. 2002, 223, U373.

7. Alam, M. S.; Naqvi, A. Z.; Kabir-ud-Din; Colloid Polym. Sci. 2007, 285, 1573.

8. Alam, M. S.; Naqvi, A. Z.; Kabir-ud-Din; J. Chem. Eng. Data 2007, 52, 1326.

9. Alam, M. S.; Naqvi, A. Z.; Kabir-ud-Din; Colloids Surf., B 2007, 57, 204.

10. Alam, M. S.; Naqvi, A. Z.; Kabir-ud-Din; J. Surfactants Deterg. 2007, 10,35 .

11. Alam, M. S.; Naqvi, A. Z.; Kabir-ud-Din; J. Colloid Interface Sci. 2007, 306, 161.

12. Kabir-ud-din; Naqvi, A. Z.; Khan, A. B.; Al-Ahmadi, M. D. A.; Akram, M.; J. Chem. Eng. Data 2009, 54, 387.

13. Chai, J. L.; Mu, J. H.; Colloid J. 2002, 64, 550.

14. Koshy, L.; Saiyad, A. H.; Rakshit, A. K.; Colloid Polym. Sci. 1996, 274, 582 . 
15. Materna, K.; Cote, G.; Szymanowski, J.; J. Colloid Interface Sci. 2004, 269,466

16. Hung, K. C.; Chen, B. H.; Yu, L. E.; Sep. Purif. Technol. 2007, 57, 1.

17. Tatara, E.; Materna, K.; Schaadt, A.; Bart, H. J.; Szymanowski, J.; Environ. Sci. Technol. 2005, 39, 3110.

18. de Oliveira, H. P.; Albuquerque, J. J. F.; Nogueiras, C.; Rieumont, J.; Int. J. Pharm. 2009, 366, 185

19. de Oliveira, H. P.; Andrade, C. A. S.; de Melo, C. P.; J. Colloid Interface Sci. 2008, 319, 441.

20. de Oliveira, H. P.; de Melo, C. P.; Synth. Met. 2006, 156, 215.

21. de Oliveira, H. P.; Oliveira, E. G. L.; de Melo, C. P.; J. Colloid Interface Sci. 2006, 303, 444.

22. de Oliveira, H. P.; Tavares, G. F.; Nogueiras, C.; Rieumont, J.; Int. J. Pharm. 2009, 380, 55.
23. de Oliveira, H. P.; Tenorio, A. C.; de Lima, E. G.; de Melo, C. P.; Colloids Surf., A 2005, 257-58, 3.

24. Lee, P. C.; Meisel, D.; J. Phys. Chem. 1982, 86, 3391.

25. Pizzitutti, F.; Bruni, F.; Rev. Sci. Instrum. 2001, 72, 2502.

26. Kohn, P.; Schroter, K.; Thurn-Albrecht, T.; Phys. Rev. Lett. 2009, 102, 216101.

27. Asami, K.; J. Non-Cryst. Solids 2002, 305, 268.

28. Kambale, R. C.; Shaikh, P. A.; Bhosale, C. H.; Rajpure, K. Y.; Kolekar, Y. D.; Smart Mater. Struct. 2009, 18, 085014.

29. Rachna, S.; Bhattacharyya, S.; Gupta, S. M.; J. Phys. Chem. Solids 2008 , 69, 822 . 